\title{
Conferencias de prensa y COVID-19: explorando la respuesta gubernamental mexicana desde la comunicación en salud
}

\section{Press conferences and COVID-19: exploring the Mexican government response from health communication}

Ninón Irene Llano Guibarraa , Julio César Aguila Sáncheza

a Doctorado en Ciencias Sociales, Universidad de Colima, México

\section{Resumen}

Introducción: La pandemia de la COVID-19 ha puesto a prueba la capacidad de los gobiernos -y de los sistemas políticos y sanitarios- para manejar situaciones excepcionales, donde la comunicación cumple un rol fundamental. Esto demanda desplegar procesos comunicativos coordinados, coherentes, asertivos y eficientes. Objetivo: El presente artículo tiene el objetivo de caracterizar las conferencias de prensa de la Secretaría de Salud de México durante la segunda fase de la pandemia de COVID-19 en el país, por ser el principal espacio de comunicación para atender la crisis. Metodología: Fueron analizadas las 28 conferencias correspondientes a la segunda fase de la pandemia a través del análisis de contenido en el programa FileMaker, versión 18. Resultados: El análisis demuestra que además de la información técnica, la información sobre la gestión gubernamental de la crisis ocupa un lugar privilegiado dentro de la conferencia. Conclusión: Este espacio de comunicación oficial tomó en consideración los elementos estructurales de vulnerabilidad que intervienen en la propagación de la pandemia, lo cual guarda una estrecha relación con el llamado a la responsabilidad individual y colectiva que hace el Estado.

Palabras clave: comunicación; conferencia de prensa; información sanitaria; salud; discurso; COVID-19; México.

\begin{abstract}
Introduction: The COVID-19 pandemic has tested the capacity of governments -and political and health systems- to handle exceptional situations, where communication plays a fundamental role. This demands the deployment of coordinated, coherent, assertive, and efficient communication processes. Objective: The purpose of this article is to characterize the press conferences of the Ministry of Health of Mexico during the second phase of the COVID-19 pandemic in the country, for being the main communication space to deal with the crisis. Methodology: The 28 conferences corresponding to the second phase of the pandemic were analyzed through content analysis in the FileMaker program, version 18. Results: The analysis shows that in addition to technical information, information on government crisis management occupies a privileged place within the conference. Conclusion: This official communication space took into account the structural elements of vulnerability that intervene in the spread of the pandemic, which is closely related to the call to individual and collective responsibility made by the State.
\end{abstract}

Keywords: communication; press conference; health information; Health; speech; COVID-19; Mexico. 


\section{Introducción}

a pandemia de COVID-19 ha puesto a prueba la capacidad de los gobiernos para manejar situaciones excepcionales. Tanto los sistemas políticos como los sanitarios se han visto afectados por la dinámica de este contexto tan complejo. El hecho de que la crisis sanitaria tenga profundas repercusiones económicas, políticas y sociales, la ha convertido en el tema de mayor cobertura en las agendas mediáticas y políticas a nivel global. El manejo de una crisis de tal magnitud exige a los gobiernos implementar estrategias de comunicación que por una parte promuevan una concientización colectiva sobre la situación; y por otra, les permita sondear -y convencer- a la opinión pública sobre su gestión.

La comunicación cumple un rol fundamental en los momentos de crisis sanitarias (Fernández de la Hoz, 2014), los cuales demandan desplegar procesos comunicativos coordinados, coherentes, asertivos y eficientes, para no crear una excesiva alarma social que desencadene un efecto rebote en la población (Silva, Garcia-Marques \& Wegener, 2019). Sobre esto, Cebrià et al. (2005) apuntan que brindar más información no promueve necesariamente actitudes más racionales en los sujetos, esto muchas veces confunde más y contribuye a la incertidumbre típica de los contextos de crisis.

Al respecto, Rodríguez (2011, p.39) señala que, "los riesgos deben ser comunicados en mensajes claros, fácilmente comprensibles, que apelen no sólo a $L a$ Razón sino también a la emoción y que no den lugar a dudas o ambigüedades". Aunque hay claridad de que promover miedo no trae consigo más cuidado de la población, continúa siendo un recurso ampliamente utilizado en la comunicación en salud (Farré, 2005). En este espacio entra en juego el debate teórico -e incluso ético- sobre su empleo, que lleva a la pregunta ¿hasta qué punto los gobiernos utilizan estratégicamente los espacios de comunicación en tiempos de crisis sanitaria?

Es importante observar que uno de los efectos de recurrir al miedo es que, pasado un tiempo, este se vuelve insostenible para los sujetos, quienes crean una barrera de inmunidad subjetiva para protegerse (Douglas, 1996). Aunado a ello, pueden comenzar a tener una selección más exquisita de las informaciones a las que se exponen. Esto no solo pasa con la población en general, también les sucede a los profesionales sanitarios que padecen el "síndrome de recorte de prensa" (Martínez, 2014) ante los procesos de metacomunicación que los rodean.

Sobre el temor al riesgo, Farré (2005) reflexiona que:

Nuestras vidas cotidianas se encuentran rodeadas por riesgos potenciales con los que convivimos voluntariamente. Fumar produce importantes riesgos para nuestra salud, circular por carretera presenta una alta probabilidad de accidentes e incluso de muerte. Este tipo de riesgo es voluntario y se encuentra integrado en nuestras decisiones del día a día de tal manera que, de tan visibles, se convierten en invisibles (p.108).

Está claro que el problema es más complejo que la decisión individual de arriesgarse o no, porque en él intervienen factores contextuales y estructurales que alejan o acercan a los sujetos del riesgo sanitario. De ahí que varios autores alertan sobre la necesidad de superar los enfoques centrados en el riesgo (Gonnet, 2011) o en el comportamiento individual de las personas (Ratzan et al., 1994; Clift \& Freimuth, 1995); y en vez de ello, proponen adoptar enfoques de vulnerabilidad (Blaikie, Cannon, Davis, \& Wisner (1996).

Este enfoque permitiría desarrollar procesos de comunicación que atiendan las condiciones estructurales subyacentes en cualquier problemática de salud (Quimbayo, Fandiño \& Jaimes, 2012) y que, al atenderlos, sean más efectivos. De acuerdo con el modelo de la Organización Mundial de la Salud, los determinantes de salud se dan en dos niveles: estructural e intermedio (OMS, 2010). Las determinantes estructurales incluyen el contexto socioeconómico y político (gobiernos, actores, políticas, mercado de trabajo, cultura y valores) y la estructura social (clase, género, edad, etnia, territorio, como ejes del poder); mientras los determinantes intermedios hacen alusión a los recursos materiales (empleos, cuidados, ingresos, vivienda y residencia). Entre esas determinantes también están los factores psicosociales, conductuales, biológicos y los servicios de salud, que en su conjunto crean desigualdades en salud (OMS, 2010). Pero ¿en qué medida los determinantes en salud, las desigualdades o vulnerabilidades, son tomadas en cuenta en la atención gubernamental a la crisis sanitaria?, o aún más: ¿en qué medida estos elementos están contenidos en los discursos y estrategias de comunicación de las instituciones? 
Para analizar los discursos de salud, Bañón (2018) propone incluir a los actores del proceso, los objetivos de las estrategias, la responsabilidad de las instituciones, la extensión, el estilo y las proyecciones de sus discursos. El autor clasifica a los actores de la comunicación en tres niveles: macroactores, mesoactores y microactores. En el primero están incluidos los encargados de las estrategias de comunicación, los responsables políticos y los representantes institucionales. En el nivel medio están los profesionales de salud, los colectivos sociales y profesionales, así como las asociaciones de profesionales sanitarios, empresas farmacéuticas e investigadores en el ámbito de la salud. Por último, a nivel micro, estarían las personas o pacientes y sus familiares.

A pesar del poco tiempo transcurrido desde el surgimiento de la pandemia, se pueden encontrar algunos estudios sobre comunicación y COVID-19 que, en ocasiones a modo de ensayo, dan luces sobre los posicionamientos y formas de acercamiento al novedoso objeto de estudio que, recordemos, sigue en curso. Peters, Jandrić y McLaren (2020) aseguran que existe una relación dialéctica entre virus y comunicación, porque ambos tienen formas de dispersión muy similares, ambos necesitan humanos para replicarse, y además son inofensivos hasta que llegan a células o lectores. $(\mathrm{Re})$ aplicando el carácter viral a la información, estos autores defienden la idea de la modernidad viral, que ha promovido una respuesta bioinformacional a la crisis actual.

Los estudios sobre redes sociales en la pandemia ocupan un lugar privilegiado hasta el momento. Wilson (2020) asegura que el pánico en las redes sociales se ha propagado más rápido que la propia COVID-19. Asimismo, el clima de desinformación e incertidumbre ha originado rumores y bulos durante la crisis (Pennycook, McPhetres, Zhang \& Rand, 2020). De esta manera, se ha visto afectada la salud mental de las personas, tanto por su exposición a las redes sociales (Gao et al., 2020), como a los medios de difusión más tradicionales (Garfin, Silver \& Holman, 2020). Estos hallazgos nos dan una idea de la importancia de desarrollar estrategias de comunicación eficientes que permitan frenar la propagación de rumores en las plataformas digitales (Cinelli et al., 2020).

Sin embargo, sobre la eficiencia comunicativa, un estudio de la narrativa del riesgo sobre COVID-19 asegura que paradójicamente en los contextos de mayor confianza pública se tiende a relajar la responsabilidad individual
(Wong \& Jensen, 2020), lo cual impone más retos a las estrategias comunicativas. En especial porque un clima de incertidumbre y desinformación -que genere miedo-, puede convertirse en el mecanismo de control social (Altheide, 2002) que necesita el Estado para manejar la crisis. Otro estudio sobre la censura y la manipulación informativa del gobierno español durante las primeras semanas de la actual crisis (Espinosa, 2020), demuestra que los medios, como antaño, siguen al servicio del Estado.

La censura y la transparencia han sido preocupaciones constantes para Occidente, especialmente en cuanto al manejo de la pandemia en China (Fu \& Zhu, 2020) e incluso sobre la actuación "pasiva" de la Organización Mundial de la Salud (OMS). La censura, la manipulación mediática y la falta de transparencia evidencian las relaciones de poder que están implícitas en todo proceso comunicativo.

Por ello, se hace pertinente adoptar enfoques interdisciplinarios en los estudios de comunicación en salud, en diálogo con otras disciplinas de las ciencias sociales, que permitan comprender las relaciones sociales contenidas en tales procesos. A tono con ello, Brown (2020) asegura que "desarrollar estrategias, narrativas y prácticas que sean inclusivas, tanto simbólica como sustantivamente (...), representa quizás la contribución más importante y tangible que las ciencias sociales críticas pueden hacer al actual proceso de gobernanza de riesgos ante la COVID-19" (p.11).

En sintonía con lo anterior, el presente artículo tiene el objetivo de caracterizar las conferencias de prensa de la Secretaría de Salud de México durante la segunda fase de la pandemia de COVID-19 en el país, por ser el principal espacio de comunicación para atender la crisis. El estudio parte del supuesto de que la retroalimentación característica de estas conferencias organizó en buena medida la agenda comunicacional del gobierno mexicano, convirtiéndose así en un "termómetro" del conocimiento general sobre el virus, del manejo de la gestión gubernamental e, inclusive, de los avatares políticos que marcaban la agenda.

\section{Método}

Las conferencias de prensa iniciaron el 29 de febrero de 2020, y a la fecha continúan su emisión por el canal de YouTube de la Secretaría de Salud y sus redes sociales. Todas las emisiones están disponibles en la página oficial (www.coronavirus.gob.mx) que el gobierno de México 
desarrolló para dar información y actualizar las estadísticas. La sección de Noticias de esta página contiene las conferencias organizadas por las fases de la pandemia, hasta la tercera (actual). Las mismas corresponden a la estrategia de "vigilancia centinela" que la Secretaría de Salud utiliza para monitorizar el desarrollo de la pandemia y las acciones a tomar. La primera fase comprende del 29 de febrero hasta el 23 de marzo, la segunda fase del 24 de marzo al 20 de abril y actualmente se desarrolla la tercera fase que comenzó el 21 de abril. Es decir, solamente están concluidas las fases 1 y 2 . Para este estudio se utilizaron como muestra las 28 conferencias de la segunda fase, en el entendido de que componen un corpus cerrado y más consolidado que la primera fase.

Después de una exploración inicial se determinó que, en términos de estrategia comunicativa, las primeras 24 conferencias de la primera fase sirvieron para que, en cierta medida, se consolidara la dinámica gubernamental de información acerca de la pandemia y se estableciera la estructura formal del espacio. Para la segunda fase se cuenta con cierta solidez que permite una aproximación analítica. Aún así, al estar la estrategia todavía en desarrollo se considera este análisis como una propuesta exploratoria. De todas formas, las 28 conferencias de la segunda fase son un nutrido campo de investigación que permite observar las dinámicas comunicativas a nivel interno y hacer preguntas sobre los efectos que estas causaron en todos los actores involucrados tanto a nivel interno como externo.

Un primer visionado de las conferencias sirvió para establecer categorías de análisis previas de acuerdo con el paradigma analítico que se plantea en la introducción de este trabajo. No obstante, se readecuaron las categorías para aprehender mejor el contexto discursivo, pero también socio-político que se manifestaba en las conferencias. A fin de contar con un instrumento homogéneo de análisis se utilizó el programa informático Filemaker (versión 18), para convertir categorías de análisis en campos de registro y así hacer un seguimiento de la presencia y evolución de aquellas. Asimismo, para contar con la información suficiente que permitiera obtener estadísticas inicialmente descriptivas. Es importante que las "categorías y variables (...) funcionen como descriptores para la caracterización" del universo estudiado (Pita González \& Grillo, 2015, p.2). De este modo, nuestra herramienta de análisis se conformó de tres apartados: estructura, temática y prensa (Tabla 1).
El apartado de estructura valora aspectos formales de presentación que se observaron en el formato de la conferencia, así como los participantes y recursos utilizados. Este apartado registra aspectos como: expositores, gráficas, recursos adicionales. Por su parte, el apartado de temática se utilizó para hacer un seguimiento de siete categorías específicas a lo largo de las 28 conferencias: temas de salud, aspectos laborales, cuestiones de género, exposición de la estrategia de gobierno, variables comparativas con el resto del mundo, exposición de diferentes vulnerabilidades de la población mexicana ante la pandemia y, finalmente, el énfasis en la responsabilidad asumida ante el contexto, ya fuera de tipo individual, colectiva, gubernamental, empresarial o mediática. Como tercero, el apartado del instrumento de análisis dedicado a la rueda de prensa se diseñó para hacer un seguimiento no sólo a los periodistas, medios de comunicación y preguntas realizadas, sino, más importante, en un intento de aproximarnos a la intencionalidad de las preguntas realizadas $y$, para equilibrar, a la preparación que demostraban los periodistas al hacer las preguntas. Cabe destacar que cada uno de los apartados incluyó un espacio para observaciones particulares que permitió profundizar en un análisis más cualitativo de cada conferencia.

Como resultado se obtuvo el llenado de 152 fichas de análisis ya que, aunque se visionó 28 conferencias, luego cada ficha también se individualizó para la participación de cada uno de los periodistas. Esto implicó que contáramos con 28 fichas correspondientes a cada una de las conferencias y además otras 124 que permitiera hacer un seguimiento puntual de la sección de rueda de prensa. Aquí es importante destacar que, después de los resultados obtenidos en el trabajo de campo, consideramos que cada uno de los apartados merecía un estudio y contextualización por separado, sin embargo, eso justifica todavía más la pertinencia de este trabajo exploratorio. En cuanto a la ejecución del trabajo de campo, se dedicó un aproximado de 2 horas y media por cada conferencia porque, aunque cada una dura una hora en su mayoría, un visionado más cualitativo exigía el detalle en el análisis y el registro de su desarrollo.

Es necesario hacer énfasis en que, si bien para los objetivos de este estudio nos limitamos a hacer un recuento estadístico de la frecuencia de repetición de las categorías expuestas, este porcentaje es un indicador cuantitativo 
en estrecha relación al seguimiento de la narrativa de toda la conferencia y del conjunto de la muestra de las 28 conferencias analizadas. Es decir, la sumatoria nos permite tener un parámetro de análisis, pero este se complementa con un análisis cualitativo del contexto y del contenido. En este sentido, es importante destacar la utilidad del instrumento de análisis en consonancia con una perspectiva de análisis de contenido que según Krippendorff (2018, p.28) es una "técnica destinada a formular, a partir de ciertos datos, inferencias reproducibles y válidas que pueden aplicarse a un contexto". De acuerdo con este autor y con nuestros objetivos de estudio, tomamos en cuenta siete elementos para analizar los datos: objetividad, sistematicidad y cuantitatividad por un lado, pero también la cualitatividad, representatividad, exhaustividad y generalización de los datos. De este modo, estos últimos nos permitieron abrir la mirada no sólo al registro de variables sino al análisis del contexto.

Finalmente, los resultados obtenidos se procesaron a través de Excel para la obtención de estadísticas iniciales y gráficos ilustrativos. Cabe destacar que una de las principales limitaciones del artículo está en que se trata de un estudio exploratorio, inicial, sobre un objeto aún en construcción, sobre y durante una crisis no superada. Lejos de intentar establecer ideas definitorias, el objetivo es que los aportes del texto contribuyan a comprender el fenómeno de estudio.

Tabla 1. Categorías y temáticas analizadas en el estudio.

\begin{tabular}{|l|l|l|}
\hline \multicolumn{1}{|c|}{$\begin{array}{c}\text { Aspectos formales de } \\
\text { estructura }\end{array}$} & \multicolumn{1}{|c|}{$\begin{array}{c}\text { Temáticas/Categorías en el } \\
\text { contenido }\end{array}$} & Rueda de prensa/periodistas \\
\hline $\begin{array}{l}\text { Expositores } \\
\text { Gráficas } \\
\text { Recursos adicionales }\end{array}$ & $\begin{array}{l}\text { Salud } \\
\text { Laboral }\end{array}$ & $\begin{array}{l}\text { Desempeño periodístico } \\
\text { Intencionalidad de pregunta }\end{array}$ \\
& $\begin{array}{l}\text { Género } \\
\text { Estrategia de gobierno }\end{array}$ & \\
& Aspectos comparativos & \\
& Vulnerabilidad & \\
& Responsabilidad & \\
\hline
\end{tabular}

Fuente: Elaboración propia. 


\section{Resultados}

Los resultados están organizados en tres subapartados: el primero sobre la conferencia como espacio de comunicación, e incluye la estructura, los participantes, la información técnica así como los recursos de comunicación empleados. El segundo es sobre los ejes temáticos principales de la conferencia, donde está todo lo relacionado con la COVID-19 como enfermedad, la estrategia gubernamental para hacer frente a la crisis sanitaria, la responsabilidad ciudadana en la pandemia y los elementos de vulnerabilidad presentes en el discurso oficial. El tercero recoge lo referente al espacio de la prensa en la conferencia, participación, tendencias de las preguntas, etc.

\section{La conferencia como espacio de comunicación}

La conferencia tiene una frecuencia diaria y se transmite desde el Palacio Nacional en Ciudad de México, al igual que las intervenciones matutinas diarias del presidente del país, quien implementó esa dinámica desde su llegada al gobierno. El espacio nació con el objetivo de informar a la población en general sobre la COVID-19 en México. Generalmente se hace una presentación de dos temas, uno fijo que es el informe técnico diario y después un tema que va variando cada día. La rueda de prensa tiene un apartado final para las preguntas de los periodistas. El presentador habitual es el subsecretario de Salud Dr. Hugo López-Gatell y además participan otros funcionarios de las instituciones de salud y de otras instancias de gobierno.

El promedio de participantes cada día es de 4 expositores en cada conferencia. Durante la segunda fase, en total hubo presencia de 6 instancias de gobierno: la Secretaría de Relaciones Exteriores, Gobernación, Defensa, Marina, el Instituto Nacional de las Mujeres y el Consejo Nacional para Prevenir la Discriminación. Solo en 4 conferencias hubo expositores de otras secretarías.
Entre los que más asistieron de la Secretaría de Salud a las 28 conferencias, además de López-Gatell que presidió casi todas (26), estuvieron el director general de Epidemiología José Luis Alomía (17), el director general de Promoción de la Salud Ricardo Cortés y el director de prestaciones médicas del Instituto Mexicano de Seguridad Social (IMSS) Víctor Hugo Borja, ambos en 8 conferencias. De las otras instancias mencionadas, los funcionarios asistieron sólo a 1 conferencia, excepto el canciller Marcelo Ebrard que asistió a 2.

El informe técnico diario detalla el panorama de la pandemia en el mundo (casos contagiados y tasas de letalidad), y en los continentes, especialmente Europa y las Américas. Después se presenta un mapa de México con la tasa de incidencia por entidad federativa, la distribución por grupos de edad y el tipo de atención médica que han recibido: ambulatoria (en casa) o con hospitalización, donde clasifican a los pacientes en estables, graves y críticos. También se presentan los datos de los recuperados y las defunciones, estasúltimasen relación a las co-morbilidades, que en el caso de México son: hipertensión, diabetes, obesidad, tabaquismo, problemas renales, enfermedad pulmonar obstructiva crónica (EPOC), inmunosupresión, asma y VIH-sida. Al final del informe técnico también se proyectaba la curva epidémica.

En la conferencia se utilizaba un lenguaje claro, con el fin de que llegue a la mayor cantidad de audiencia. Aunque empleaban tecnicismos propios del campo de la salud como, por ejemplo, cuando hablaban de diabetes gestacional explicaron que son "cambios en el azúcar en las embarazadas" o cuando se refirieron a la carga viral, aclararon que es la "concentración de virus en una muestra de sangre". Para representar los datos, además de la gráficas y mapas, se emplearon imágenes (54\%), vídeos (25\%), aplicaciones de teléfonos móviles (11\%) y páginas web (11\%) (Figura 1).

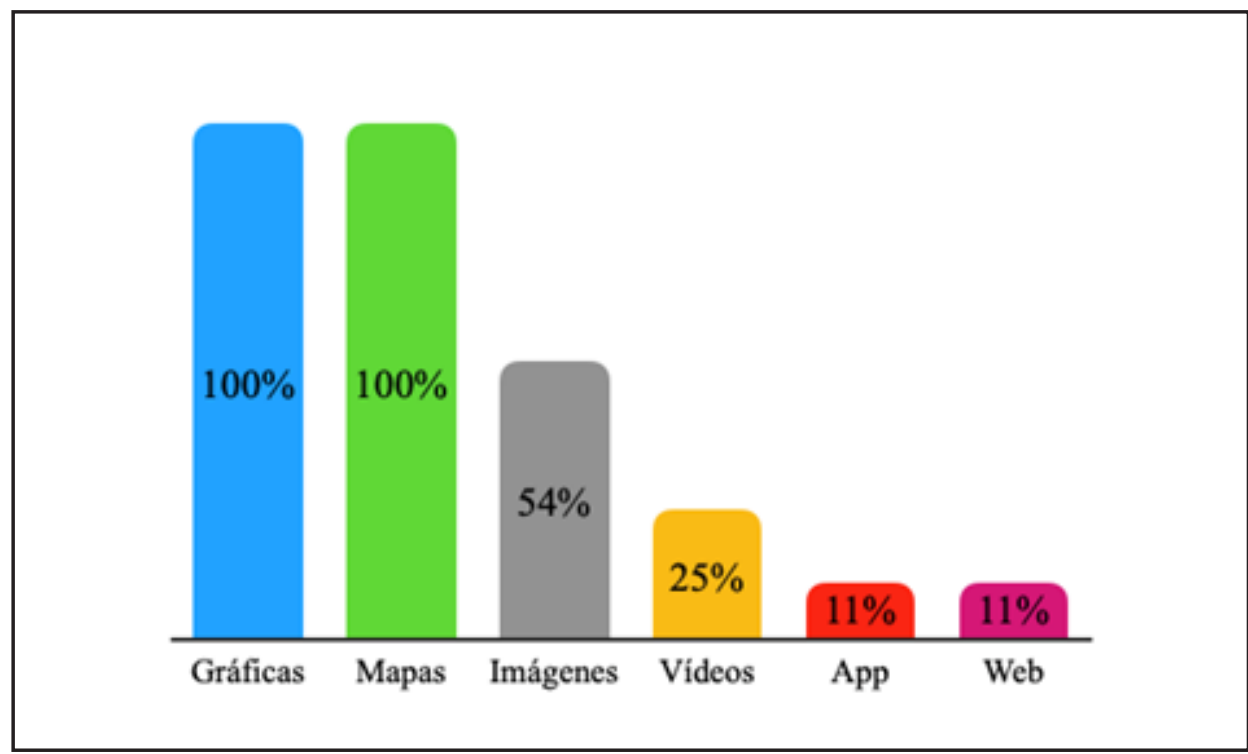

Figura 1. Recursos comunicativos empleados. Fuente: Elaboración propia. 
También se utiliza como un espacio para transmitir mensajes y recomendaciones a la población sobre las acciones de cuidado que se deben implementar en la emergencia sanitaria. En las conferencias se encontró una combinación de mensajes entre los de corte racional $(32 \%)$ con los emocionales (21\%). Un ejemplo de racional fue cuando explicaban la importancia de las donaciones de sangre: "el principal incentivo es que debemos donar sangre porque todos podemos necesitarla en algún momento" o cuando explicaban cuánto de contagioso es el virus. $Y$ en el caso de los emocionales, se encontró: "piense en un ser querido, quédese en casa". También recurrían a la repetición de frases como "quédate en casa" en la mayoría de las conferencias (71\%), y a la interpelación del otro, es decir, mensajes dirigidos directamente a la audiencia, como, por ejemplo: "tú que te querías ir de vacaciones" o "usted que me está viendo desde casa".

\section{Los ejes temáticos de la conferencia}

Entre los temas de salud más tratados estaba lo relacionado con las co-morbilidades y los factores de riesgo, que pueden agravar la situación de los pacientes, lo cual es un tema de interés público, por los niveles de diabetes, hipertensión y obesidad en la población mexicana (Soto-Estrada, Moreno-Altamirano,
\& Pahua, 2016). Se menciona, asimismo, la evolución de los casos por entidades federativas y la diferenciación entre coronavirus y COVID-19, además de las recomendaciones para el cuidado de los ciudadanos, así como comparaciones de México con el mundo (32\%), o con países de Europa (57\%), con Estados Unidos (36\%) y con los de América Latina (21\%).

La conferencia ha sido un espacio no solo para informar sobre la enfermedad, sino para explicar la estrategia que ha adoptado el gobierno para manejar la crisis sanitaria. Por ello, hubo una amplia presencia de disertaciones sobre las estrategias de contención (25\%) y mitigación (39\%), así como la Jornada Nacional de Sana Distancia (64\%). También sobre la estrategia de comunicación en salud (46\%) y el manejo de insumos: gestiones y abastecimiento de material sanitario $(50 \%)$ y saturación de hospitales $(36 \%)$ principalmente. Aquí sobresale el vínculo entre atención a la COVID-19 y el brote de influenza (21\%) que padeció México en 2009, cuya infraestructura de atención, ahora es reorientada para la crisis actual. También aparecieron disposiciones gubernamentales firmadas para la emergencia sanitaria como: las garantías laborales para los empleados (25\%) en general y para los trabajadores de salud (39\%) en particular (Figura 2).

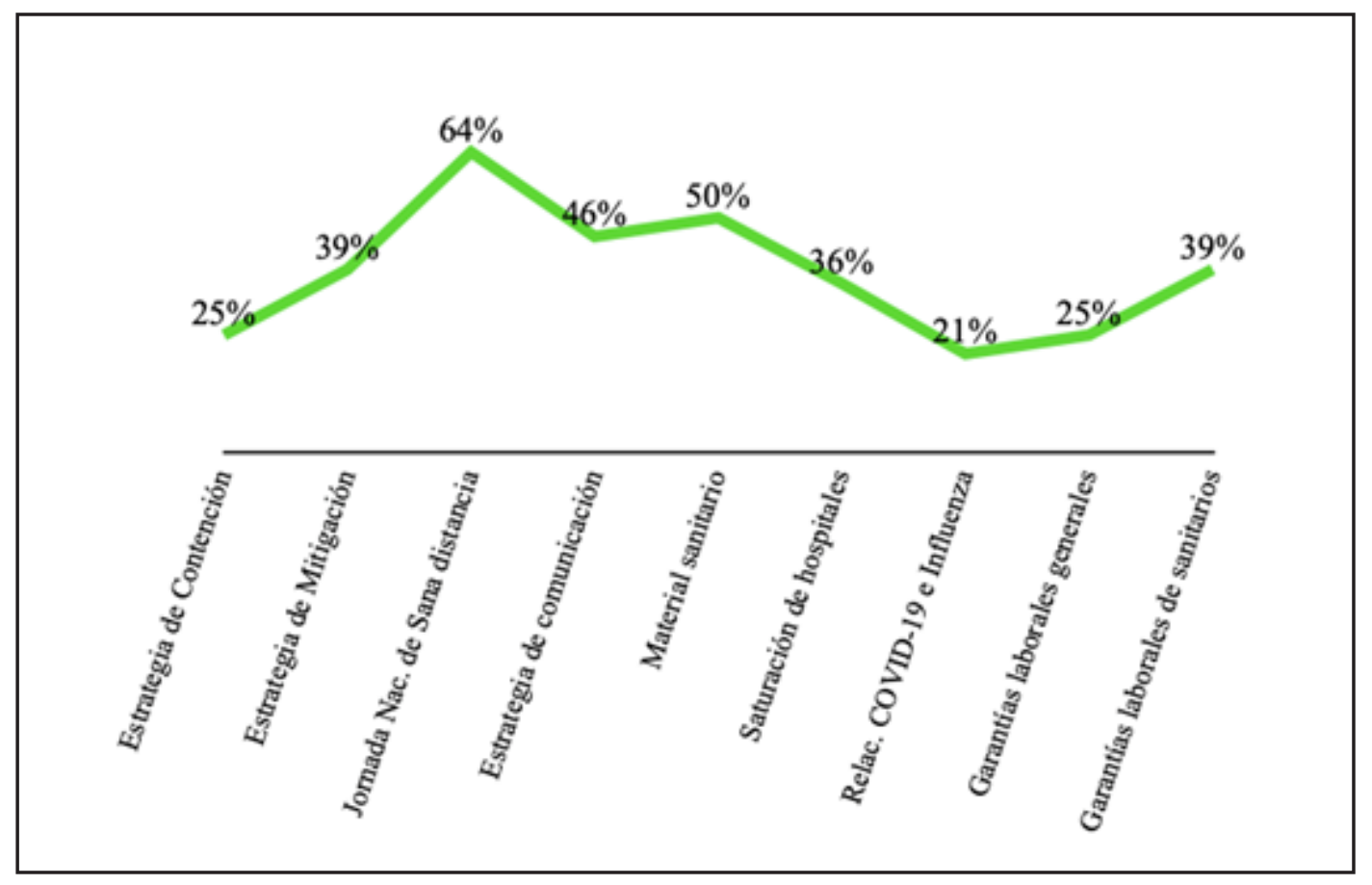

Figura 2. Principales temáticas de la conferencia . Fuente: Elaboración propia 
Una estrategia de comunicación en salud en una pandemia exige responsabilidad de todos los niveles. En todas las conferencias se trató el tema de las responsabilidades, con este orden de repetición: colectiva (54\%), gubernamental $(43 \%)$, individual $(43 \%)$, mediática (32\%) y empresarial (21\%). Otro tema interesante fue el tratamiento a la relación COVID-19 y vulnerabilidad. La salud y la enfermedad no se distribuyen de forma homogénea en la población, hay quienes están más expuestos al riesgo de enfermarse y hay quienes tienen más protegida su salud. $Y$ esto no tiene que ver exclusivamente con los factores de riesgo biológico.

Entre las vulnerabilidades que más se trataron en la conferencia estuvieron: la biológica, que hace alusión a la edad o las comorbilidades (54\%), la laboral (47\%), especialmente de los sanitarios, la económica (25\%), la geográfica: por zona de residencia rural y urbana (29\%), el acceso a la información (18\%), la de género (14\%) y la étnica (14\%). Resulta interesante lo poco que se habla de estas dos últimas, respecto del resto, en un país con altos índices de violencia contra los indígenas (HurtadoSaa, Rosas-Vargas, \& Valdés-Cobos, 2013) y contra las mujeres (CEPAL, 2019) (Figura 3).

En cuanto al género como vulnerabilidad, entre los temas tratados resaltaron: la violencia de género como problema de salud pública de larga data en México, la responsabilidad de los medios en la relación entre violencia, riesgo y mujeres, así como los servicios de atención a las mujeres durante la crisis.

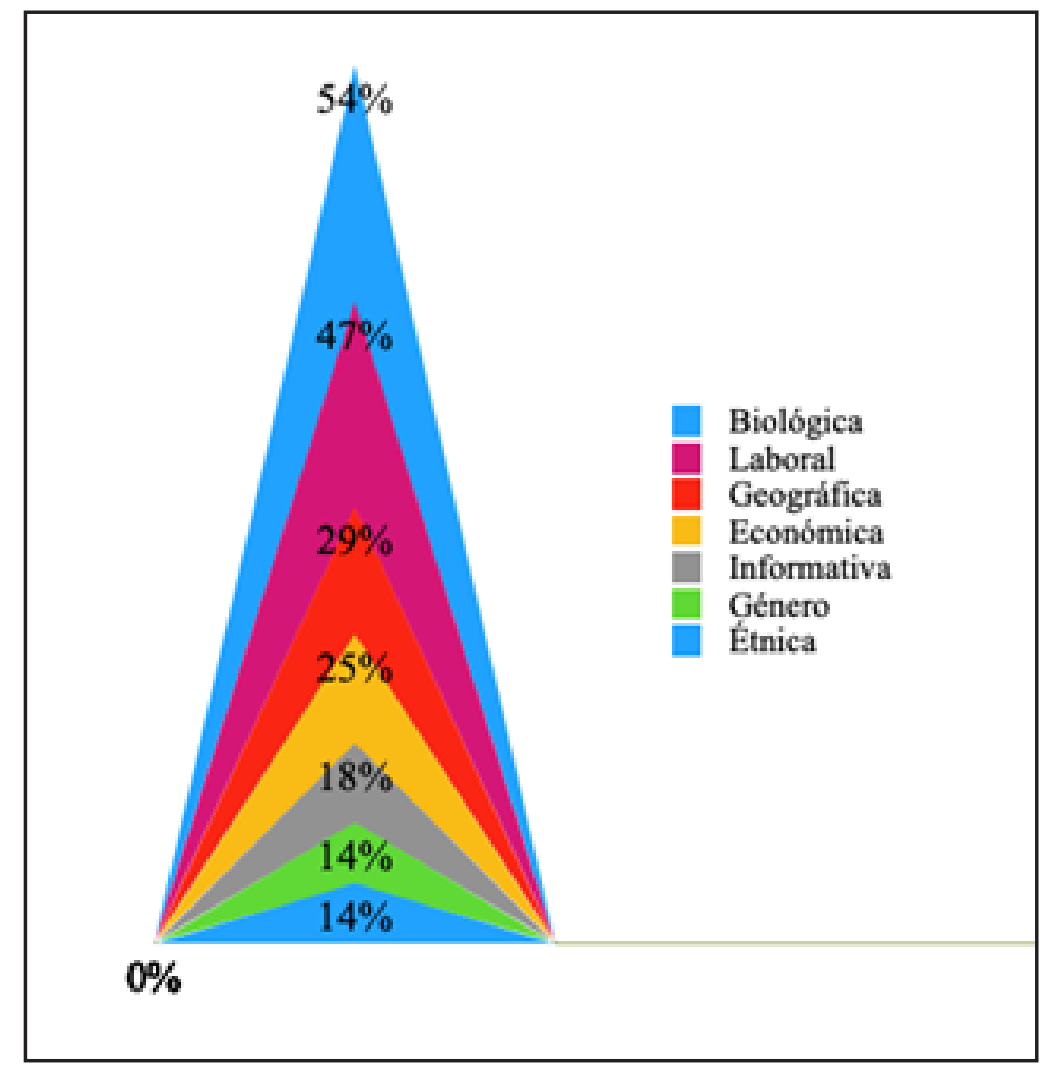

Figura 3. Vulnerabilidades más representadas. Fuente: Elaboración propia. 


\section{La prensa}

Al final de la presentación se dejaba un espacio para las preguntas de los periodistas. En cada conferencia preguntaron un promedio de cinco reporteros y los medios más representados fueron: Milenio (16\%), Notimex (15\%) y La Jornada (13\%) (Figura 4).

En cuanto al desempeño periodístico, el $63 \%$ se ayudaba de una guía para hacer sus preguntas, el $66 \%$ de las preguntas tenían relevancia social, entre las que destacan: ¿Recomiendan algún medicamento para los que están enfermos en casa? ¿Cómo se determina el alta de una persona recuperada? ¿En qué momento entramos en la fase 3 , qué condiciones habrá? ¿Cuánto va a aumentar la pobreza de México? En ocasiones hacían preguntas reiterativas $(24 \%)$, una que salió varias veces fue ¿las personas que tienen que salir porque realizan trabajos esenciales, tendrán que usar cubrebocas? o sobre el empleo de personal sanitario ¿si nos puede recordar cuáles son los números de la meta de contratación tanto de médicos como de enfermeras y si tienen una fecha límite para alcanzar ese nivel de reclutamiento?

También se encontraron preguntas no relacionadas (30\%) con el tema de la conferencia de ese día, sin aprovechar que tenían presente a funcionarios que podían aclarar dudas sobre las acciones de la institución que representaban. Por ejemplo, el día 26 de marzo que asistieron a la conferencia Nadina Gasman, directora del Instituto Nacional de las Mujeres, Karla Berdichevzky, directora del Centro Nacional de Equidad de Género y Salud Reproductiva y Lorena Rodríguez, del Consejo Nacional de Salud Mental, no preguntaron nada relacionado con el tema de la conferencia: convivencia en casa y género en la crisis.

Solo el $32 \%$ solicitó aclaración sobre los datos o estadísticas expuestas por los conferenciantes. Para sustentar sus preguntas, un $17 \%$ hizo referencia a noticias de fuentes no contrastadas, incluso a supuestas informaciones en las redes sociales. También fue evidente una impersonalización (17\%) de la pregunta, es decir, no la hacían a nombre suyo, sino como una supuesta inquietud poblacional. En general hicieron muy pocas preguntas de especialización (13\%) en el área de la comunicación en salud.

Entre las preguntas de la prensa, el $45 \%$ estaba encaminada a información sobre la enfermedad (formas de transmisión, recomendaciones de cuidados, hallazgos, tratamientos, etc.), como, por ejemplo: ¿Los estimados deben estar en aislamiento? ¿Qué pasa con los pacientes que ya son como dados de alta? ¿Uno de los síntomas es la pérdida de olfato, la diarrea, no sé si esto es cierto o no, si usted nos podría platicar? ¿Se ha informado que se utilizará plasma para tratar a los pacientes, tienen información al respecto? ¿Cuándo podremos tener una vacuna?

El 40\% tenía el objetivo de indagar sobre la gestión gubernamental de la crisis, con preguntas como: ¿Cuánto se está invirtiendo y cuántos protocolos hay en México para la cura de la COVID-19? ¿Si se desbordan los hospitales, qué va a pasar con los que no tengan cama? ¿Tienen contempladas medidas más severas para que la población se mantenga en casa? ¿Qué le dice a usted de que los gobernadores tomen decisiones por su parte y de que incluso se hable de que haya un grupo de gobernadores que quieran hacer un bloque independiente para tomar sus propias medidas?

Otras preguntas estuvieron encaminadas a las estadísticas $(12 \%)$ manejadas o sobre peticiones $(3 \%)$ de incluir determinadas aclaraciones o desglose de estadísticas en próximas conferencias. También resaltó el sensacionalismo de la prensa en preguntas como: “¿Nos podría dar un estimado de cuántos fallecimientos habrá? ¿decenas, miles o millones? digo para tranquilizar a la gente" (Figura 5).

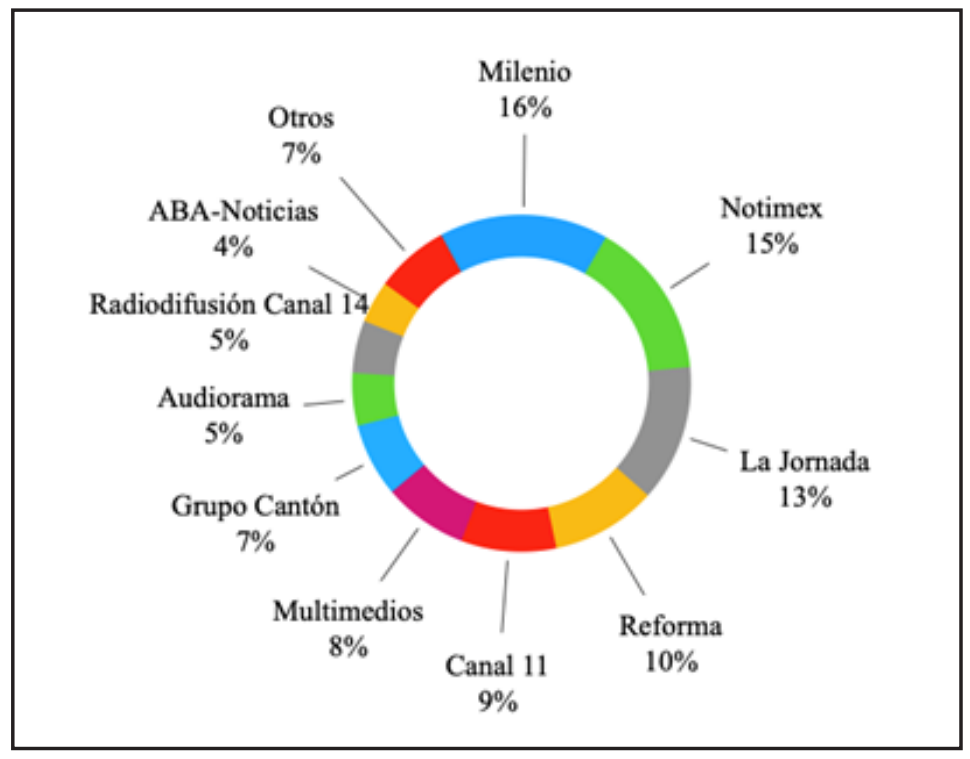

Figura 4. Medios de comunicación más representados. Fuente: Elaboración propia 


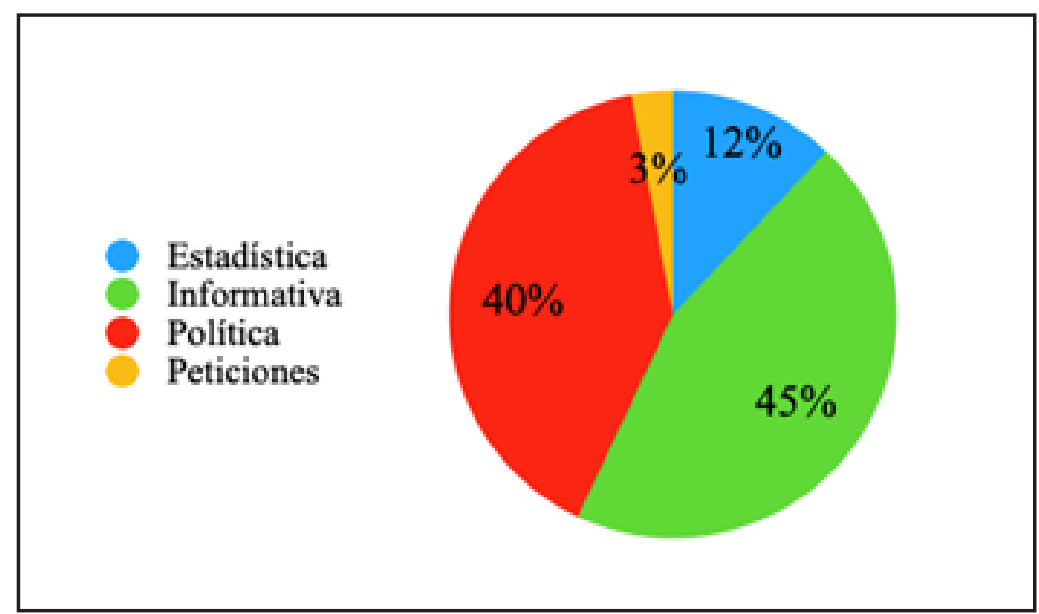

Figura 5. Intencionalidad en las preguntas de los reporteros. Fuente: Elaboración propia.

\section{Discusión}

Los medios de comunicación tienen "una notable influencia sobre la salud pública", por ser una de las principales fuentes de información de la población sobre temas de salud, incluso "por delante de los profesionales sanitarios" (Catalán-Matamoros, 2015, p.217). Esto demanda determinadas habilidades persuasivas para la transmisión efectiva del mensaje. Al respecto, apunta Nabi (2017) que "tendencias recientes de la investigación sobre narrativas persuasivas y mensajes virales sugieren que la emoción puede jugar un papel clave en la persuasión basada en los medios".

Sin embargo, una línea argumentativa racional ayuda a crear climas de confianza en la población sobre la gestión de las crisis. Señalan Wong y Olivia $(2020$, p.9) que "la confianza es un componente esencial de lo que mantiene unida a la sociedad, especialmente en tiempos de crisis". Para esto es necesario contar con autoridad, validación social, reciprocidad, escasez, simpatía y coherencia (Cialdini, 2001). De ahí que el protagonismo del subsecretario de Salud, así como la persuasión de la estrategia, hayan sido clave en las conferencias.

Puede decirse que las mismas siguen una línea argumentativa racional, porque explican los motivos y las causas de las medidas tomadas o propuestas. Además, sus decisiones las argumentan y comparan con hechos y datos del accionar gubernamental de otros países (Petty, Cacioppo, \& Goldman, 1981). Entre los principales argumentos racionales de la conferencia para recomendar quedarse en casa, están los siguientes: las formas de propagación del virus, la necesidad del distanciamiento social y de otras medidas como el constante lavado de mano, el uso y abuso de mascarillas o cubrebocas en espacios públicos,que, en ese momento, no eran obligatorias para salir a la calle porque "no está probado -científicamente- que ayuden a disminuir el riesgo".
Tampoco es un mensaje impositivo, sino una recomendación, "si puede quédese en casa", eso hace que sean más convincente, porque no parece una obstinación absurda del Estado para confinarlos. Esto, unido a la cita de fuentes y el análisis de la actuación de otros países, ayudan a una mayor credibilidad del emisor. Por otra parte, aparece la estrategia de los mensajes con sentido común, más apegados a la realidad, al reconocer que "no todos se pueden quedar en casa". Como apuntan Gutiérrez y Blázquez (2016, p.160): "si deseamos tener una relación más cercana, es fundamental humanizar nuestra comunicación", lo cual queda más evidente en los espacios que ha creado la conferencia, por ejemplo, para dialogar con el personal sanitario, en específico de enfermería, a raíz de los eventos de agresiones en hospitales del país.

Estos contenidos de la conferencia, los estilos de comunicación, así como su organización y diseño, además de lo apresurado que ha sido, son parte de las estrategias de seducción (Lasen \& Martínez, 2008) de la comunicación para concientizar a la población del peligro y a la vez gestionar la opinión pública sobre la gestión gubernamental de la crisis sanitaria. Además de que no se han tenido en cuenta algunos elementos, precisamente por la premura de la contingencia, lo cual no es una peculiaridad exclusiva del caso mexicano, sino global. Como apuntan Balog-Way y McComas (2020, p.1): "aunque la COVID-19 fue inevitablemente 'inesperado', se pasaron por alto importantes acciones de preparación, como la creación de capacidades clave de comunicación de riesgos". Aquí cabría preguntarse ¿hasta qué punto los gobiernos construyen de forma constante una estrategia de comunicación específicamente destinada a situaciones excepcionales, de riesgo o de desastre?

Entre los temas tratados era importante hablar de las comorbilidades en México, por el peso que tienen en el empeoramiento de la situación de salud de 
quienes padecen el virus. Por ejemplo, la obesidad es un problema de salud pública en México desde hace varias décadas (Torres \& Rojas, 2018) y junto con las infecciones respiratorias agudas, la diabetes mellitus tipo 2, el asma y las enfermedades pulmonares como la neumonía e influenza, figuran entre las principales causas de mortalidad y de morbilidad del país (SotoEstrada, Moreno-Altamirano \& Pahua, 2016). Tratar esos temas en la conferencia era parte del rescate de una problemática de antaño que se hace vigente en la situación actual.

Sucede lo mismo con el tema de la pobreza, cuando López-Gatell afirmó que viene de políticas económicas de varias décadas (Secretaría de Salud, 2020). Sin embargo, también estos discursos sirven para legitimar el gobierno actual, respecto de los anteriores gobiernos mexicanos y respecto de otros gobiernos del mundo por su actuación ante la pandemia. En eso ayudan las comparaciones que aparecen en la conferencia, por ejemplo, con Europa y Estados Unidos. También se puede ver el discurso sobre la actual infraestructura del gobierno mexicano para atender la influenza, que se ha readecuado en función de la COVID-19, y aparece como una de las fortalezas que promueven confianza en el sistema, o que, en todo caso, sirven para legitimar una gestión que -como otras gestiones a nivel mundial- puede pasar por improvisada.

En la actual pandemia, según Pennycook, McPhetres, Zhang, y Rand (2020, p.14) "ha habido cierta polarización política en el contexto de COVID-19". $Y$ es que las crisis sanitarias no escapan a las contradicciones políticas, de hecho, pueden ser motivo para la legitimidad o deslegitimidad política (Kvarnlöf \& Montelius 2020). La crisis está poniendo a prueba las capacidades del gobierno mexicano y de todos los gobiernos del mundo y sacando a la luz problemáticas sociales de antaño. Quizá esta sea la prueba de fuego del gobierno mexicano para hacer valer su insignia propagandística de la campaña electoral que rezaba "primero los pobres", al ser la comunicación de la gestión de la crisis un "termómetro" para medir la atención de la desigualdad social por parte del Estado.

Las crisis no sirven solo para legitimar a los gobernantes, sino a sus formas de gobernanza. Un estudio sobre el cólera de 1865 señala que la llegada de esta enfermedad ayudó "a legitimar la autoridad y las relaciones de dominio, siendo un factor estabilizador en el medio y largo plazo" (Salas \& Pujadas-Mora, 2016, p.212). $Y$ añaden los autores que:

La persistencia de epidemias, reales 0 invisibles, en un mundo cada vez más globalizado, reforzó la idea de que el camino adecuado para superar este tipo de episodios pasaba ineludiblemente por aumentar la vigilancia, el control social -especialmente la pobreza- y la progresiva medicalización de la sociedad (Salas \& Pujadas-Mora, 2016, p.211).

Agregan los autores que el cólera "ayudó a crear una identificación avalada por la ciencia más clara entre los teóricos 'agentes' causales y de la epidemia y determinados sectores de la población. En este sentido, higienismo y contagionismo se daban otra vez la mano (...), identificando los productores de miseria, los pobres, dados también a los mayores e inmorales 'excesos" (Salas \& Pujadas-Mora, 2016, p.211). De esta manera, la legitimación oficial de determinadas ideas, en este caso la pobreza, produce una legitimación de la marginalización social que padecen estos grupos.

Y ahí es donde está la importancia de que el llamado no sea a la responsabilidad individual, sino colectiva, gubernamental, empresarial, mediática. Porque un llamado a lo individual no reconocería que muchos individuos se arriesgan a salir de casa, no porque sean irresponsables, sino porque no les queda otro remedio que ir a trabajar para subsistir. Es el caso de los cincuenta millones de pobres (CONEVAL, 2018) que hay en México y en donde el $60 \%$ de los trabajadores son informales (OIT, 2014).

Como apunta Zinn (2020, p.6): "dar prioridad a las medidas epidemiológicas para controlar la propagación de un virus mortal como el bloqueo integral de la vida social afecta a los grupos sociales de manera desigual y produce riesgos secundarios". De ahí la importancia de tener en cuenta esta desigualdad del impacto de las medidas y por supuesto, la manera en que se tratan estos temas en los discursos oficiales y en los medios de comunicación. Apuntan Giritli y Olofsson $(2020$, p.5) que:

Ya hemos sido testigos de cómo las personas en posiciones bastante privilegiadas son las que tienen la capacidad de trabajar desde casa, lo que significa que también tienen más potencial para actuar de acuerdo con las recomendaciones de salud, mientras que otros corren el riesgo de ser despedidos de su trabajo o de sus negocios en quiebra.

Estos son los elementos que no pueden faltar en una estrategia de contención y mitigación de una pandemia como la COVID-19 en un país con tanta desigualdad (Ugalde, 2018). Además, hacer gala de ella en sus discursos, y no solo en el discurso estatal, sino también en las agendas de los medios de comunicación, velar por estos contenidos, ponerlos en la agenda pública, también es parte del trabajo de la prensa. Evidentemente la prensa mexicana ha estado más interesada en la gestión gubernamental que en la enfermedad en sí, aunque no 
siempre con la intención de hacer una crítica constructiva a la gestión, tampoco podría decirse que con el fin de deslegitimarla, sin embargo, sí han demostrado poca preparación en la comunicación en salud, lo cual guarda estrecha relación con la escasez de especialización del área en México y en muchos países del mundo.

Muchas veces se han dejado llevar más por la búsqueda de un titular impactante, con preguntas sensacionalistas y falta de fundamentos, como parte de esa ola de infodemia que inunda a México como el segundo país con más falsas noticias del mundo (Fragoso, 2018). Se impone un reto mayor a la gestión de comunicación en la crisis sanitaria (Hurtado, 2020), en medio de esa perspectiva bioinformacional de la atención global de la pandemia que hablan Peters, Jandrić y McLaren (2020).

\section{Conclusiones}

Las conferencias de prensa de la Secretaría de Salud de México durante la segunda fase de la pandemia de COVID-19 en el país, se caracterizan por desarrollar un lenguaje claro y preciso, para una mejor comprensión de los temas en la población. En relación a ello, apunta Catalán-Matamoros (2015, p.222) que "cuando falta claridad y abundan los términos técnicos, difícilmente comprensibles por el público, se limita la recepción de la información". Asimismo, las conferencias se han convertido en un espacio no solo para informaciones técnicas sobre salud/enfermedad y el caso puntual del desarrollo de la pandemia de COVID-19 en el país, sino también en un espacio para la presentación y debate público de las cuestiones de vulnerabilidad y desigualdad social que influyen en la salud de la población. Esto es particularmente importante por el contexto de desigualdad que acontece en la región latinoamericana.

El claro interés en incluir en su agenda y hacer énfasis no solo en las medidas individuales sino en explicar las medidas que va tomando el gobierno en función de atender la contingencia sanitaria, evidencia que las conferencias de prensa como espacio de comunicación oficial de la actual crisis, tienen el objetivo de incidir en la opinión pública sobre la gestión del gobierno, a la vez que concientizar a la población de la situación de riesgo. En esto influyen los recursos persuasivos que utilizan, así como las estrategias para generar confianza -ante todo política- en la gestión de la crisis.

De esta manera se está poniendo a prueba la capacidad de gestión del gobierno mexicano, no solo en la cuestión sanitaria o del sistema de salud, sino en una gestión política integral que relacione la salud con la economía, el empleo, el género, la etnia, el lugar de residencia, la violencia o el acceso a la información, entre otros elementos que intervienen en ella.

Esto demuestra que la retroalimentación característica de estas conferencias organizó en buena medida la agenda comunicacional del gobierno mexicano, convirtiéndose así en un "termómetro" del conocimiento general sobre el virus, del manejo de la gestión gubernamental e, inclusive, de los avatares políticos que marcaban la agenda. A partir de este estudio inicial cabría preguntarse ¿hasta qué punto la dinámica interactiva afecta positiva o negativamente a la política comunicacional?, ¿el debate político termina influyendo en una suerte de ruido comunicacional?, o por el contrario ¿contribuyen a cuestionar saludablemente la legitimidad de un gobierno?, ¿es este el espacio adecuado para ello? y, ya en términos más generales ¿es posible que el espacio para la comunicación en salud sirva también para generar debate y conciencia acerca de las condiciones estructurales a nivel social?

\section{Financiación}

Ambos autores son becarios doctorales (2016-2020) del Consejo Latinoamericano de Ciencias Sociales y el Consejo Nacional de Ciencia y Tecnología de México.

\section{Referencias bibliográficas}

Altheide, D. L. (2002). Creating fear. News and the Construction of Crisis. New York: Aldine de Gruyter.

Balog-Way, D. H. P., \& McComas, K. A. (2020). COVID-19: Reflections on trust, tradeoffs, and preparedness. Journal of Risk Research, in press, 1-11. https:// doi.org/10.1080/13669877.2020.1758192

Bañón, A. M. (2018). Discurso y salud. Análisis de un debate social. Pamplona: Ediciones Universidad de Navarra.

Blaikie, P., Cannon, T., Davis, I., \& Wisner, B. (1996). Vulnerabilidad: el entorno social, político y económico de los desastres. Bogotá, Colombia: La Red ITDG.

Brown, P. (2020). Studying COVID-19 in light of critical approaches to risk and uncertainty: research pathways, conceptual tools, and some magic from Mary Douglas. Health, Risk \& Society, 22(1), 1-14. https://doi.org/10.1080/13698575.2020.1745508

Catalán-Matamoros, D. (2015). Periodismo en salud: análisis de los públicos, formatos y efectos. Panacea, 16(42), 217-224. Recuperado de: https://bit.ly/2Tbw1rr 
Cebrià, J., Palma, C., Gutiérrez, I., Ferrer, M., Ger, S., \& Doménech, M. (2005). Estudio del grado de aprensión social en usuarios de atención primaria: su relación con la ansiedad. Atención Primaria, 36(5), 254-260. Recuperado de: https://doi.org/10.1157/13079147

Cialdini, R. B. (2001). The science of persuasion. Scientific American, 284, 76-81. https://doi. org/10.1038/scientificamerican0201-76

Cinelli, M., Quattrociocchi, W., Galeazzi, A., Valensise, C. M., Brugnoli, W., Schmidt, A. L., ... Scala, A. (2020). The COVID-19 Social Media Infodemic. Physics and Society, arXiv:2003.05004v1. Recuperado de https://arxiv.org/abs/2003.05004

Clift, E., \& Freimuth, V. (1995). Health Communication: What Is It and What Can It Do for You? Journal of Health Education, 26(2), 68-74. Recuperado de http://bit.ly/2TRI91j

Comisión Económica para América Latina y el Caribe. (2019). América Latina, el Caribe y España (19 países): Feminicidio o femicidio, último año disponible (En números absolutos y tasas por cada 100.000 mujeres). Recuperado de: bit.ly/2QgUiut

Consejo Nacional de Evaluación de la Política de Desarrollo Social. (2018). Pobreza en México. Recuperado de: https://bit.ly/2WZck75

Douglas, M. (1996). La aceptabilidad del riesgo según las ciencias sociales. Barcelona, España: Paidós.

Espinosa, N. (2020). Censura y manipulación informativa durante las primeras semanas de la crisis del Coronavirus en España. La Razón Histórica, 46(1), 39-62. Recuperado de: https://bit.ly/3coFLWZ

Farré, J. (2015). Comunicación de riesgo y espirales del miedo. Comunicación y Sociedad, 2(3), 95-119. https://doi.org/10.32870/cys.v0i3.4209

Fernández de la Hoz, K. (2014). La comunicación en crisis sanitarias: perspectiva de las administraciones públicas. Revista Española de Comunicación en Salud, 5(1), 14-19. Recuperado de https://bit.ly/2L9qIJW

Fregoso, J. (2018). \#Mexico2018|'Fake news'and Social Media: The New Heads of the Hydra. University of Oxford. Recuperado de: https://bit.ly/2T7mm5d

Fu, K., \& Zhu, Y. (2020). Did the world overlook the media's early warning of COVID-19? Journal of Risk Research, 2-5. https://doi.org/10.1080/13669 877.2020 .1756380
Gao, J., Zheng, P., Jia, Y., Chen, H., Mao, Y., Chen, S., ... Dai, J. (2020). Mental health problems and social media exposure during COVID-19 Outbreak. PLoS ONE, 15(4), 1-10. https://doi.org/10.1371/journal. pone.0231924

Garfin, D. R., Silver, R. C., \& Holman, E. A. (2020). The Novel Coronavirus (COVID-2019) Outbreak: Amplification of Public Health Consequences by Media Exposure. Health Psychology, 39(5), 355357. http://dx.doi.org/10.1037/hea0000875

Giritli, K., \& Olofsson, A. (2020). Managing the Covid-19 pandemic through individual responsibility: the consequences of a world risk society and enhanced ethopolitics. Journal of Risk Research, in press, 1-5. https://doi.org/10.1080/13669877.2020.1756382

Gonnet, J. P. (2011). Las distintas conceptualizaciones del Riesgo en la Sociología. Riesgo como consecuencia de la modernidad reflexiva, como consecuencia de la modernidad y como estructura universal de todo orden social. Akrópolis Umuarama, 19(3), 175185. http://doi.org/10.25110/akrópolis.v19i3.4031

Gutiérrez, J. M., \& Blázquez, A. (2016). Confianza emocional y racional en marketing político. Dimensión Empresarial, 14(1), 151-161. http:// dx.doi.org/10.15665/rde.v14i1.575

Hurtado, L. A. (2020, abril 9). Además de pandemia por COVID-19, México enfrenta propagación de noticias falsas. Boletin_UNAM-DGCS. Recuperado de: https://bit.ly/3bCoYON

Hurtado-Saa, T., Rosas-Vargas, R., \& Valdés-Cobos, A. (2013). Servicios de salud, discriminación y condición étnica/racial: un estudio de caso de la problemática en México y Colombia. Ra Ximhai, 9(1), 135-151. https://doi.org/10.35197/rx.09.01.e.2013.12.th

Krippendorff, K. (2018). Content Analysis. An Introduction to Its Methodology. 4th ed. California, Estados Unidos: Sage Publications.

Kvarnlöf, L., \& Montelius, E. (2020). Militariseringen av Covid-19. Dagens Arena. Recuperado de https:// bit.ly/2Z8QyjX

Lasen, A., \& Martinez, I. (2008). Movimientos, "mobidas" y móviles. Un análisis de las masas mediatizadas. En A. I. Sádab, \& Á. Gordo (Coords.), Cultura digital y movimientos sociales (pp. 243-266). Madrid, España: Catarata. 
Martínez, P. (2014). ANIS: Conciliación de intereses entre informadores y comunicadores de la salud. Revista Española de Comunicación en Salud, 5(1), 2427. Recuperado de https://bit.ly/3cemPK7

Nabi, R. L. (2017). Emotion in Media Persuasion. En P. Rössler, C. A. Hoffner, \& L. van Zoonen (Eds.), The International Encyclopedia of Media Effects (pp. 1-8). Hoboken, NJ, USA: John Wiley \& Sons, Inc. https://doi.org/10.1002/9781118783764.wbieme0161

Organización Internacional del Trabajo. (2014). El empleo informal en México: situación actual, políticas y desafíos. Recuperado de: https://bit. ly/2ySvuDU

Organización Mundial de la Salud. (2010). A Conceptual Framework for Action on the Social Determinants of Health. Recuperado de https://bit.ly/2zwavHj

Pennycook, G., McPhetres, J., Zhang, Y., \& Rand, D. G. (2020). Fighting COVID-19 misinformation on social media: Experimental evidence for a scalable accuracy nudge intervention. PsyArXiv, 1-24. https:// doi.org/10.31234/osf.io/uhbk9

Peters, M. A., Jandrić, P., \& McLaren, P. (2020). Viral modernity? epidemics, infodemics, and the 'bioinformational' paradigm. Educational Philosophy and Theory, 1-23. http://doi.org/10.10 80/00131857.2020.1744226

Petty R. E., Cacioppo J. T. Y., \& Goldman. R. (1981). Personal Involvement as a Determinant of ArgumentBased Persuasion. Journal of Personality and Social Psychology, 41(5), 847-855. http://doi. org/10.1037/0022-3514.41.5.847

Pita González, A., \& Grillo, M. (2015). Una propuesta de análisis para el estudio de revistas culturales. Revista Latinoamericana de Metodología de las Ciencias Sociales, 5(1), 1-30. Recuperado de https://bit.ly/2ZHWNvt

Quimbayo, L. R., Fandiño, V. C. \& Jaimes, L. M. (2012). Condiciones de vida desde el enfoque de los determinantes sociales en salud en un grupo organizado de madres adolescentes. Revista Cuidarte, 1, 308-319. http://doi.org/10.15649/ cuidarte.v3i1.26

Ratzan, S., Stearns, N. S., Payne, J. G., Amato, P. P., Liebergott, J. W., \& Madoff, M. A. (1994). Education for the Health Communication Professional. American
Behavioral Scientist, 38(2), 361-380. http://doi.or $\mathrm{g} / 10.1177 \% 2 \mathrm{~F} 0002764294038002015$

Rodríguez, R. (2011). La efectividad del uso del miedo como factor persuasivo en la comunicación de riesgos en las crisis sanitarias. Revista de Comunicación y Salud, 1(2), 33-46. https://doi.org/10.35669/revist adecomunicacionysalud.2011.1(2).33-46

Salas, P., \& Pujadas-Mora, J. M. (2016). El cólera como conflicto y factor de legitimación. Palma 1865. Ayer, 101(1), 189-212. Recuperado de https://bit.ly/2zCFj8S

Secretaría de Salud. (26 de marzo de 2020). Conferencia de prensa sobre COVID-19. [Archivo de vídeo]. Recuperado de: https://bit.ly/3fOuhxQ

Silva, P. P., Garcia-Marques, T., \& Wegener, D.T. (2019). Efectos rebote en la persuasión: una consideración de los posibles efectos irónicos de la supresión y la corrección. Revista de Psicología Social, 34(3), 575589. https://doi.org/10.1080/02134748.2019.1649962

Soto-Estrada, G., Moreno-Altamirano, L., \& Pahua, D. (2016). Panorama epidemiológico de México, principales causas de morbilidad y mortalidad. Revista de la Facultad de Medicina (México), 59(6), 8-22. Recuperado de: https://bit.ly/3cBBLIW

Torres, F., \& Rojas, A. (2018). Obesidad y salud pública en México: transformación del patrón hegemónico de oferta-demanda de alimentos. Problemas del Desarrollo, 49(193), 145-169. https://dx.doi. org/10.22201/iiec.20078951e.2018.193.63185

Ugalde, V. (2018). Desigualdades y justicia social: informe Desigualdades en México 2018. Estudios demográficos y urbanos, 33(3), 799-804. https:// dx.doi.org/10.24201/edu.v33i3.1882

Wilson, M. E., \& Chen, L. H. (2020). Travelers Give Wings to Novel Coronavirus (2019-nCoV). Journal of Travel Medicine, 27(2), 1-3. https://doi. org/10.1093/jtm/taaa015

Wong, C. M. L., \& Jensen, O. (2020). The paradox of trust: perceived risk and public compliance during the COVID-19 pandemic in Singapore. Journal of Risk Research, 1-10. http://doi.org/10.1080/13669 877.2020.1756386

Zinn, J. O. (2020). 'A monstrous threat': how a state of exception turns into a 'new normal'. Journal of Risk Research, in press, 1-9. https://doi.org/ 10.1080/13669877.2020.1758194 